\title{
Necrotising MRSA pneumonia with mycotic pulmonary artery pseudo - aneurysm in an infant - ready to pop out!!!
}

Natasha Gupta $^{1}$, ARPITA CHATTOPADHYAY ${ }^{1}$, Rutuja Kishor Nyayadhish ${ }^{1}$, and Diganta Saikia ${ }^{1}$

\author{
${ }^{1}$ Chacha Nehru Bal Chikitsalaya
}

November 30, 2020

\author{
Abstract \\ pop out!!! \\ Dr. Natasha Gupta, MD (First author) \\ Specialist (Dept of Radiology) \\ Chacha Nehru Bal Chikitsalaya, Geeta Colony, \\ New Delhi. \\ Email id: drnatashagupta@gmail.com \\ Dr. Arpita Chattopadhyay, MD (Corresponding author) \\ Assistant Professor (Dept of Pediatrics) \\ Chacha Nehru Bal Chikitsalaya, Geeta Colony, \\ New Delhi. \\ Email id: chattopadhyay.arpita@gmail.com \\ Dr. Rutuja Kishor Nyayadhish, DCH \\ Postgraduate Trainee (Dept of Pediatrics) \\ Chacha Nehru Bal Chikitsalaya, Geeta Colony, \\ New Delhi. \\ Email id: rutuja.0709@gmail.com \\ Dr. Diganta Saikia, DCH, MD \\ Professor (Dept of Pediatrics) \\ Chacha Nehru Bal Chikitsalaya, Geeta Colony, \\ New Delhi.
}

Necrotising pneumonia complicated by mycotic pseudoaneurysm has been reported very rarely in infancy. Here we present a five month old girl who had an acute life-threatening presentation of cavitatory pneumonia.

Necrotising MRSA pneumonia with mycotic pulmonary artery pseudo - aneurysm in an infant - ready to 
Email id: drsaikiadiganta@gmail.com

Funding: none

Conflict of interest: none

Necrotising MRSA pneumonia with mycotic pulmonary artery pseudo - aneurysm in an infant - ready to pop out!!!

Natasha Gupta ${ }^{1}$, Arpita Chattopadhyay ${ }^{2}$, Rutuja Kishor Nyayadhish ${ }^{3}$, Diganta Saikia ${ }^{4}$

Corresponding author: Dr. Arpita Chattopadhyay, Assistant Professor (Pediatrics), Chacha Nehru Bal Chikitsalaya, Geeta Colony, New Delhi. Chattopadhyay.arpita@gmail.com

${ }^{1}$ Specialist (Radiology), ${ }^{2}$ Assistant Professor (Pediatrics), ${ }^{3}$ Postgraduate Trainee (Pediatrics) ${ }^{4}$ Professor (Pediatrics)

Affiliation: Chacha Nehru Bal Chikitsalaya, Geeta Colony, New Delhi.

To the Editor,

Necrotising pneumonia complicated by mycotic pseudoaneurysm has been reported very rarely in infancy. Here we present a five month old girl who had an acute life-threatening presentation of cavitatory pneumonia.

A five month old girl presented with painful swelling and induration over right lower limb, below the knee joint, associated with high grade fever for 15 days. For this complaint she was taken to a local practitioner where she was advised incision and drainage of underlying collection along with a course of intravenous antibiotics. However, although the limb swelling subsided, high grade fever persisted. She progressively developed rapid breathing over 5 days and presented to our tertiary care centre in respiratory failure.

Examination revealed a sick child with respiratory rate $-74 / \mathrm{min}$, HR- $186 / \mathrm{min}$, saturation $81 \%$ on room air along with severe suprasternal and subcostal retractions, diminished breath sounds over right hemithorax. Laboratory indices suggested neutrophilic leukocytosis with high C-reactive Protein. A Chest X Ray was obtained (Fig 1A), which showed multiple large sized thin walled, cystic lesions in entire right lung field and left lower zone in retrocardiac region, suggestive of pneumatoceles, with the largest lesion in the right lower lung showing an eccentric rounded mass within the cavity, projecting inside from its medial wall (Figure 1).

Due to impending respiratory failure, child was emergently intubated after shifting to the PICU and put on mechanical ventilation. After 2 hours of mechanical ventilation child suddenly began to desaturate despite titration in settings. A repeat x-ray revealed a large area of relative lucency with absent bronchovascular markings in the right hemithorax, suggesting a pneumothorax. An emergent rightside intercostal drain was inserted. The drain emptied $100 \mathrm{ml}$ of purulent fluid in the next three days. Subsequently over the course of five days, the child was gradually weaned off mechanical ventilation and shifted to humidified high flow nasal cannula. Blood culture came positive for Methicillin Resistant Staphylococcus aureus sensitive to Vancomycin and Linezolid.

As tachypnea persisted, a contrast enhanced CT scan of the Chest was done, which showed multiple thin walled, rounded to irregular, air filled cavities seen in right upper, right middle and left lower lobes, the larger lesion in right middle lobe measuring $5.3 \times 2.8 \times 3.2 \mathrm{~cm}$, causing indentation of right heart border. Another large cavitatory lesion was seen in the right lower lobe, with evidence of mild consolidatory changes in the surrounding lung parenchyma. An intensely enhancing lobulated rounded to ovoid lesion measuring 2.1 X1.5 $\mathrm{X} 2.0 \mathrm{~cm}$ was noted along the medial wall of this cavity, projecting into its lumen, which showed enhancement, analogous to vascular enhancement in the mediastinum. This lesion was seen to directly communicate with a branch from descending branch of right pulmonary artery. A subcentimeter well defined nodular lesion was seen in the parenchyma of left upper lobe posteriorly, with a small peripheral subsegmental branch of pulmonary artery seen to enter the nodule (Positive feeding vessel sign), s/o Septic embolus in the lung, in view of the already diagnosed Staphylococcal sepsis. A small fluid collection was also seen in right pleural 
cavity, with thickened enhancing pleura, s/o small empyema, with residual foci of air in pleural cavity from the previous intervention (Fig $2 \& 3$ ).

Findings were suggestive of multiple pneumatoceles in both lungs, septic embolus in left upper lobe and pulmonary artery pseudoaneurysm arising from the descending branch of right pulmonary artery.

All the above findings can be explained as sequelae to Methicillin Resistant Staphylococcal sepsis in the infant. Pneumatoceles may have developed due to disseminated bacterimic seeding, while repeated septic emboli to pulmonary arterial tree may have caused destruction and weakening of the wall of vessel, resulting in a saccular outpouching from the vessel wall, forming a pseudoaneurysm ${ }^{1}$. Though rare, acquired pulmonary artery aneurysms have been reported in adults with necrotising MRSA pneumonia, however, there is only one report in 7 month old infant who underwent successful embolization of peripheral pulmonary artery aneurysm ${ }^{2}$.Mycotic aneurysms are mostly reported in patients with infective endocarditis, intravenous drug abuse, traumatic injury and sepsis due to Staphylococcus, Streptococcus or Mucormycosis.

In view of the potentially life-threatening nature of this condition, in the probable event of rupture of the pseudoaneurysm, the baby was referred to a higher centre for interventional management. He developed one episode of hemoptysis during his stay and was subsequently planned for high risk coil embolization which maybe a challenge considering the small age of the patient, or lobectomy if it fails.

\section{LEGENDS}

Fig 1- $\mathrm{X}$ rays of the Chest taken in $\mathrm{AP}$ view in supine position before and after placement of right sided intercostal drainage tube (ICD).

Both X rays show multiple thin walled, rounded, air filled cystic lesions in both lungs (yellow arrows), s/o Pneumatoceles. A well defined soft tissue density lesion is seen projecting into the cavity in right lower lung from its medial wall (red arrows).

In Fig 1A (Pre ICD insertion)- Surrounding the pneumatoceles is an area of relative lucency, which appears to be completely devoid of lung markings (black arrow). Opacity is seen along the right lateral chest wall and in the region of right costo-phrenic angle (white arrow). These findings are s/o presence of both fluid and air in the pleural cavity in the Chest $\mathrm{X}$ ray of the patient taken in the supine position.

Fig 1B (Post ICD insertion)- There is marked reduction in the amount of air in right pleural cavity, while no change is appreciated in the appearance of pneumatoceles or the intracavitary soft tissue mass.

\section{Fig 2- 2D CECT Images}

Fig 2A- CECT Chest- 2D MPR (Multiplanar Reconstructions) in axial, coronal and sagittal sections in the mediastinal window setting show an intensely enhancing lobulated lesion (red arrow), with similar density as mediastinal vessels, located within the medial part of cavity in right lower lobe (yellow arrow). Lesion is seen to arise from branch of descending branch of right pulmonary artery on the sagittal image (black arrow) Small amount of pleural fluid collection with thickened enhancing pleural layers (white arrow) is also noted in right hemithorax, s/o small empyema.

Fig 2B- MPR sections in axial and coronal planes in lung window setting reveal multiple thin walled cavities in both lungs (Right $>$ Left), s/o Pneumatoceles (red asterix). Small nodular lesion with positive feeding vessel sign is noted in left upper lobe, s/o Septic embolus (double black arrows).

\section{Fig 3- 3D CT Reconstructions}

Fig 3A- 3D MinIP (Minimum Intensity Projection) images in axial \& coronal planes depict multiple thin walled air filled pneumatoceles in both lungs (yellow arrows)

Fig 3B- 3D MIP (Maximum Intensity Projection) images and Fig 3C- 3D VR (Volume Reconstructed) images (viewed from anterior and posterior aspects) depict intensely enhancing lobulated eccentric lesion 
(red arrow) arising from descending branch of right pulmonary artery (white arrow), s/o Pulmonary Artery Pseudoaneurysm.

\section{References:}

1. Koneru H, Biswas S, Monirul I, Hesham A, Bandyopadhyay D, Madan N , et al. Pulmonary Artery Pseudoaneurysm: A Rare Cause of Fatal Massive Hemoptysis. Case Reports in Pulmonology, vol. 2018, Article ID 8251967, 4 pages, 2018.

2. Martin BJ, Stephanie H, Muddassir R, Brent MI, Benjamin A, Peter B, Martin G. Successful embolization of an infected aneurysm of a subsegmental pulmonary artery in an infant with necrotizing MRSA pneumonia. Radiol Case Rep. 2019 Jul 4;14(9):1079-1083.
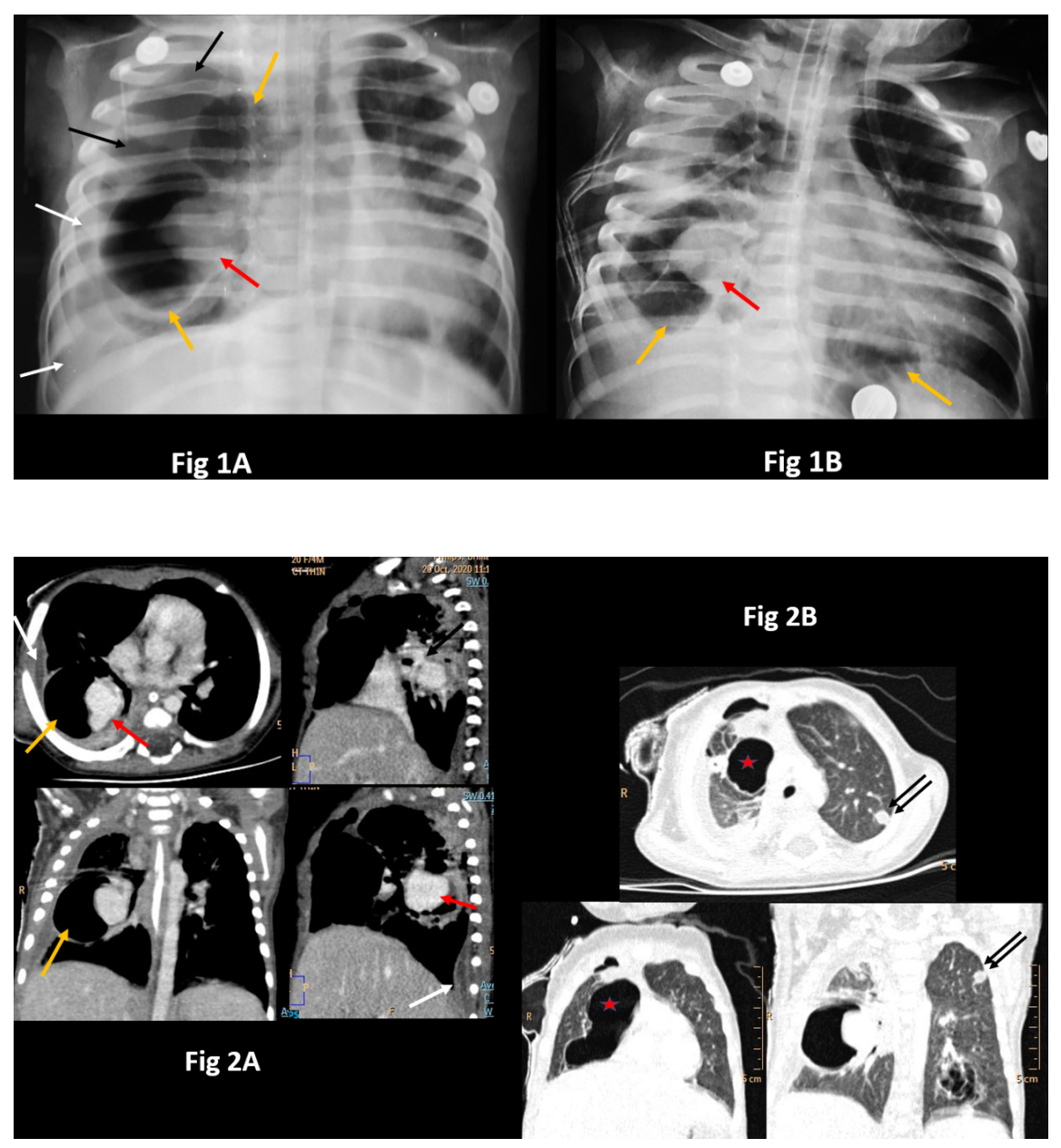


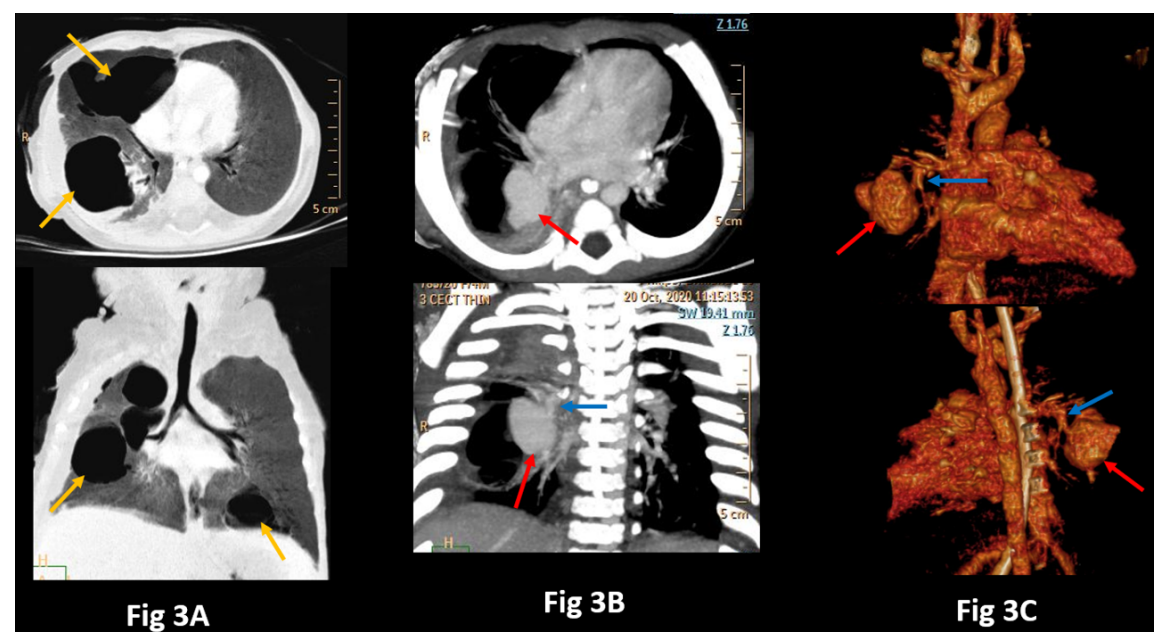

